\title{
Absorption and emission properties of QD-like particles
}

\author{
Maxim Gladush ${ }^{1,2}$ and Ekaterina Smirnova ${ }^{1,3, *}$ \\ ${ }^{1}$ Institute for Spectroscopy RAS, 108840 Troitsk, Moscow, Russia, \\ ${ }^{2}$ Moscow State Pedagogical University, 119435 Moscow, Russia \\ ${ }^{3}$ Moscow Institute of Physics and Technology, 142700, Dolgoprudny, Moscow region, Russia
}

\begin{abstract}
In this work we present a theoretical calculation of the absorption spectra of a weak probe signal and the resonance fluorescence spectra of two-level systems with a cw excitation with phonon-induced transitions.
\end{abstract}

Resonance fluorescence is the phenomenon of light emission by quantum systems in an external field. Specific spectrum known as Mollow triplet was firstly observed from $\mathrm{Na}$ gas [1] and later from organic molecules [2]. Today quantum dots are being studied as objects that demonstrate resonance fluorescence. In the latest experiments the resonance fluorescence including asymmetric Mollow triplets were observed [3,4].

In this study we calculated the spectra of the collective ensemble of two-level emitters. It was considered that in a dense homogeneous ensemble in a laser light each atom is driven by an effective field. The work [5] was taken into account to consider the collective relaxation rate. A characteristic feature of the fluorescence spectrum of this system is that the side peaks in the Mollow triplet are spectrally moved from the center.

To simulate a resonance fluorescence spectrum of a single QD the model of quantum emitter was considered with the mechanisms of quantum transitions between its states and processes of cross-relaxation of coherences due to the electron-phonon interaction. The possibility of asymmetric Mollow triplet was demonstrated. The assumptions about the model of quantum dot were taken from work [4].

We carried out simulations of resonance fluorescence spectra and the probe signal absorption spectra of an ensemble of QD-like particles and demonstrated the possibility of the appearance of an asymmetric Mollow triplet with positions of the side peaks strongly dependent on laser detuning from the frequency of the transition in the emitter. This result qualitatively corresponds to the spectral dependences recorded in experiments with single quantum dots.

This research was supported by Russian Science Foundation (№ 17-72-20266).

\section{References}

1. F. Y. Wu, R. E. Grove, S. Ezekiel, Phys. Rev. Lett., 35, 1426 (1975)

\footnotetext{
* Corresponding author: ksmirno@yandex.ru
} 
2. G. Wrigge et al., Nature Physics, 4, 60 (2008)

3. Y. J. Wei et al., Phys. Rev. Lett., 113, 097401 (2014)

4. A. Ulhaq et al., Opt. Express, 21, 4382 (2013)

5. M. G. Gladush, D. V. Kuznetsov, V. K. Roerich, Eur. Phys. J. D, 64, 511 (2011) 